\title{
PATIENT AND PERSONNEL HEALTH AND SAFETY IN MAGNETIC RESONANCE IMAGING FACILITY
}

\author{
M. Israel ${ }^{1,2^{*}}$, M. Ivanova², P. Ivanova ${ }^{2}$, Ts. Shalamanova ${ }^{2}$, H. Petkova ${ }^{2}$ \\ ${ }^{1}$ Medical University - Pleven, Bulgaria \\ 2 National Centre of Public Health and Analyses, Sofia, Bulgaria
}

\begin{abstract}
There are two aspects to be considered when using magnetic resonance imaging (MRI) equipment for diagnosis: patient and personnel protection. In regard to patient protection, the following main issues should be treated: individual characteristics, risk / benefit ratio; exposure time and exposure pattern, etc. The medical personnel protection is regulated by Directive 2013/35/EU and represents a major challenge in the EMF protection in the working environment. The Directive recognizes that for some activities/circumstances related to the installation, testing, use, development, maintenance and research related to magnetic resonance imaging (MRI) equipment may not comply with the exposure limit values. In these cases, the regulatory document introduces derogations that provide for risk management approaches for that specific source. This paper presents results of electromagnetic field measurement and evaluation in various MRI units in Bulgaria. The results show that the exposure limits for persons at specific risk are exceeded, as well as high values of the magnetic flux density of the static magnetic field up to $351 \mathrm{mT}$ in the shielded room are registered. It should be noted here that for the personnel, a serious problem is the movement in inhomogeneous field conditions (in the shielded room), which in turn leads to induction of currents in the human body and, as a result, to transient symptoms such as vertigo and nausea occur. Measurement data are used to evaluate personnel exposure and to make specific recommendations for health and safety when dealing with such equipment in medical practice.
\end{abstract}

Keywords: MRI, exposure assessment, legislation, health, protection

\section{INTRODUCTION}

Magnetic resonance imaging (MRI) is one of the most commonly used methods in diagnostic practice. It can be used to obtain images of any part of the body, providing better soft tissue contrast than computed tomography (CT). MRI can distinguish different media in the body: water, fat, muscle, etc., providing very useful and detailed information for the diagnosis of various diseases and conditions without involving ionizing radiation.

There is no hospital in Bulgaria without one or more MRI devices, which are often used in conjunction with computed tomography. More than $80 \%$ of the used MRI systems operate at $1.5 \mathrm{~T}$, but there are also single MRIs with 3 T magnets.

In recent years an increase of using the clinical application of complex hybrid machines such as LINAC MRI (MRI guided linear accelerator) and PET-MR (positron emission tomography - magnetic resonance imaging) is observed. They combine the advantages of using both non-ionizing and ionizing radiation in diagnostic procedures.
The wide application of MRI in medical practice raises the issue for protection both of patients and a large contingent of medical personnel who apply and guide this method and non-medical staff associated with the maintenance of considered devices.

The biological effects considered for the patients and personnel in MRI are result of exposure to static magnetic fields, pulse gradient fields and radiofrequency magnetic fields emitted by the equipment.

The main biological effects considered $[1,2,3,4]$ are direct effects of the static magnetic field, related to the presence of electrolytes in the blood, effects on the paramagnetic and diamagnetic components of tissues and the effects of Lorentz forces, as well as those caused by motion in inhomogeneous magnetic field. When moving in static magnetic field, the induced electric field in the head can cause dizziness and other sensory perceptions such as nausea, visual sensations (magnetophosphenes) and a metallic taste in the mouth. Other possible effects are neurocognitive effects, such as changes in attention, concentration, and visual-spatial orientation; stimulation of peripheral nerves $[2,4]$. These effects can be a cause

*michelisrael@abv.bg 
for anxiety, impaired performance, including accidents at work.

There are no data in the scientific literature on serious effects on human health from the effects of static magnetic fields up to $8 \mathrm{~T}$, but it should be noted that so far there still not enough data from epidemiological studies of long-term effects. Studies of Kromhout et al. and Borges et al. [5, 6] show development of hypertension after long-term exposure to static magnetic fields among workers from a MRI device manufacturing facility.

The gradient fields used in MRI can lead to the induction of time-varying electric fields and currents in biological tissues, which in turn could lead to stimulation of excitable tissues - peripheral nerves and muscles.

Radio frequency fields are considered in connection with the possible heating of biological tissues. The report presents results of measurement and evaluation of the electromagnetic field of seven 1.5 T MRI devices in various hospitals and medical centres in the country. In almost all medical units in our country 1.5-3 T MRI devices are used, so our research is focused on them. It should be mentioned that presented results do not reflect the highest possible exposure of the personnel around MRI. Nevertheless, these values above the ELV/ALs are not expected, but still there is a risk of high exposures to people at specific risk, as well as in the cases of movement inside the shielded room and projectile risk.

The measurements of the electromagnetic field (EMF) around the MRI were made according to a methodology developed for this purpose. The EMF exposure assessment of the personnel whose work is directly related to MRI was performed on the basis of Directive 2013/35/EU [5], and for the work places accessible to the general public according to the reference levels specified in Council Recommendation 1999/519/EC [6]. The specific risks for patients and MRI staff are considered. The results of EMF measurement in the shielded MRI rooms are summarized.

\section{MATERIAL AND METHODS}

Seven MRI devices operating at $1.5 \mathrm{~T}$ on the territory of the country during the last year were studied. Types of the studied devices were as follows: Siemens Magnetom Aera; one Siemens Magnetom Amira; one Siemens Magnetom Symphony; one Philips Achieva; one Philips Latham Magnet; one General Electric Signa HDxt Excite. The size of shielded rooms is between $30-40 \mathrm{~m}^{2}$.

The measurements were performed in the shielded MRI room, as well as in the control room. The measuring points are selected at different distances from the bore on both sides of the scanner table where the personnel can stay during standard MRI procedures: at $20 \mathrm{~cm}, 50 \mathrm{~cm}, 1 \mathrm{~m}$, at the boundary of the controlled access area and others where the personnel has specified that activities are being carried out. The measurements were performed on three levels relative to the floor of the room in order to assess the inhomogeneity of the magnetic field in the vertical. Spatial averaging for places with highly non-uniform exposure conditions according to the directive is not correct. So the exposure assessment is conservative i.e. on the basis of the maximal measured value from the three levels above the ground at the position of the body position of workers.

In all cases, the selection of measurement points took into account the procedures where there is a risk of exceeding the relevant exposure limit values (ELVs) for gradient field exposures during the various MRI studies (Table 2 from Non-binding guide to good practice for implementing Directive 2013/35/EU Electromagnetic Fields, Volume 1: Practical Guide).

According to the guide [9], the procedures with medium and high risk are as follows:

- medium risk: General anaesthesia (close monitoring of patient condition during scanning); Cardiac stress test (close monitoring of patient condition during scanning); Cleaning / infection control inside scanner (no scanning); Comforting child during scanning (comforter remains outside scanner, but within $1 \mathrm{~m}$ of aperture);

- high risk: Guide wire placement (with real time scanning); Interventional techniques such as interventional cardiovascular MRI; Functional MRI (in-scanner physical stimulation of patient); Adjustment of EEG electrodes (research activity).

During the measurements, a phantom of the human body - head, is placed in the MRI. It is made in order to ensure standard conditions. A standard protocol of MRI operation with corresponding pulse sequences is specified with the personnel to be used during the measurement. Gradient field were measured during unified sequences from the following groups: spin-echo ( $\mathrm{T} 1$ weighted, $\mathrm{T} 2$ weighted), Inversion recovery (Flair) and diffusion weighted (Conventional DWI).

All measurements were performed in an airequivalent environment, without the presence of an operator at all workplaces. During the measurements, the isotropy characteristic of the measuring equipment was taken into account in order to choose the correct approach in the measurement procedure.

The exposure assessment of personnel whose work is directly related to MRI was performed on the basis of Directive 2013/35/EU [7], transposed into national legislation by Ordinance No RD-07-5/15.11.2016 [10].

In accordance with the requirements of Directive 2013/35/ EU [7] for persons at specific risk (pregnant, wearing active and passive implants), as well as for the work places accessible to the general public, exposure assessment is performed according to the reference levels specified in Council Recommendation 1999/519/EC [8]. Here, persons whose activities are not directly related to MRI procedures are considered as general public.

\subsection{Measurement equipment}

The measurements were performed using the following measurement equipment:

- Holaday Industries HI 3550, USA, for static magnetic field o $\mathrm{Hz}$;

- Holaday Industries HI3604, with anisotropic probe, frequency range $20 \div 1000 \mathrm{~Hz}$;

- Holaday Industries HI 3603, with anisotropic probe, frequency range $8 \div 300 \mathrm{kHz}$; 
- Narda Safety Test Solutions EMR 21C, Germany, for RF electric field, frequency range $100 \mathrm{kHz} \div 3 \mathrm{GHz}$.

Expanded uncertainty for each frequency range is about $30 \%$. In the uncertainty budget, the uncertainty of calibration of measurement device, of frequency dependence of the sensor, of anisotropy of the probe, methodological uncertainties are included.

\subsection{Exposure limit values/Action \\ Values/Reference Levels}

The exposure limits used for the assessment are according to the Directive/Ordinance $[7,10]$ and Council Recommendation 1999/519/EC [8]. They are as follows:

Directive 2013/35/EC/Ordinance - ELV for static magnetic field $8 \mathrm{~T}$ for controlled working conditions; ALs $0.5 \mathrm{mT}$ for active implanted devices; $3 \mathrm{mT}$ for projectile risk; ALs are depending on the measurement frequency range for the frequencies between $300 \mathrm{~Hz}-10 \mathrm{MHz}(100 \mathrm{kHz}-1 \mathrm{MHz})$.

Council Recommendation 1999/519/EC - basic restriction and reference level for static magnetic field $40 \mathrm{mT}$; Reference values depending on the measurement frequency range for the frequencies between $0.8 \mathrm{kHz}-1 \mathrm{MHz}$. (150 kHz).

\section{RESULTS}

The results of measurements in seven MRI premises 1.5 T magnets are presented. The MRI devices are of the same type $(1.5 \mathrm{~T})$ and have been tested by our team in developing a method for measurement and exposure assessment of EMF. Here, only the results of measurements in the procedure (shielded) room are presented. The experience from our measurements so far shows that in the control room, when the procedure room is properly shielded, values more than $20 \%$ of the action levels of the time varying electromagnetic fields are not registered. The magnetic field levels in the control room depend on the placement of the equipment in control room and type of magnet's shielding.

The results of the measurements are presented in the following figures. On each of them on the abscissa the 7 examined MRIs, and on the ordinate the maximal measured values of magnetic flux density are presented for the respective frequency /frequency range at the considered distances around the bore. The results from measurements of static magnetic fields for the 7 studied MRI devices are presented on a quartile diagram as well for the three distances from the bore.

\subsection{Static magnetic field}

The measured values of magnetic flux density of the static magnetic field are presented in Figure 1 . Distribution of the static field magnetic flux densities for the seven MRI on three distances from the bore are presented in Figure 2.

Here, on quartile diagram median values from all measurements on particular distance from the bore of MRI devices and the most deviated values from the median are presented. As it could be seen from Fig. 2 on distance $1.0 \mathrm{~m}$ from the bore the median of measured flux densities is approximately equal to the median of measured values on $0.5 \mathrm{~m}$. This is due to the different angle of projection of the measuring point relative to the bore entrance.

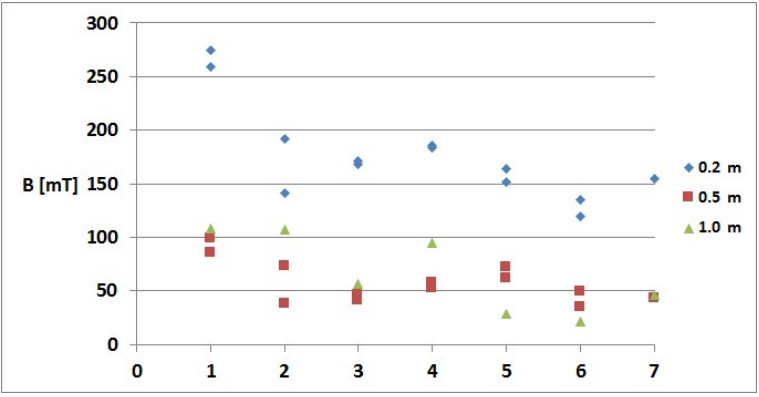

Figure 1. Measured values of the static field magnetic flux density, for the seven MRI devices

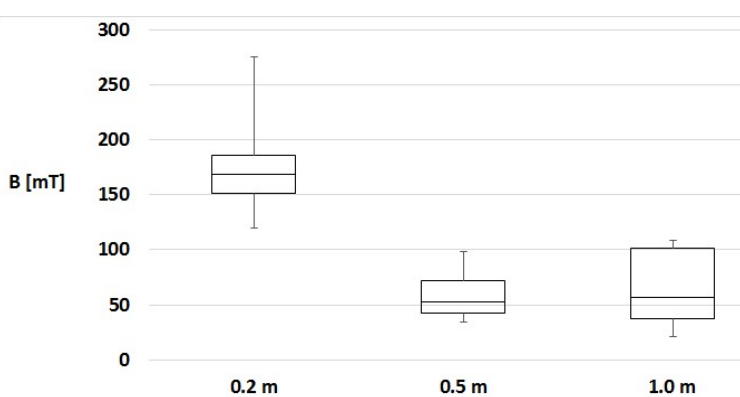

Figure 2. Distribution of the static field magnetic flux densities for the seven MRI on three distances from the bore

The results of the measurements of the static magnetic field show inhomogeneity in both the horizontal and vertical planes. When measuring at different levels relative to the floor of the room, differences from $50 \mathrm{mT}$ to $200 \mathrm{mT}$ are found between the values of the magnetic field at the level of the head and at the level of the pelvis of the workers. The maximum measured values reach $351 \mathrm{mT}$. They are registered near the entrance of the bore.

\subsection{Extremely low and low frequency magnetic fields}

The measurements of the magnetic flux density of the extremely low and low frequency magnetic fields are presented in Figure 3.

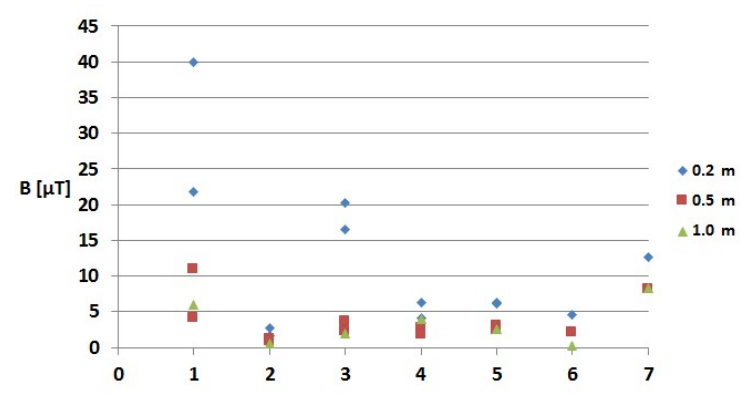

Figure 3. Measured values of the magnetic flux density in the range $20 \div 1000 \mathrm{~Hz}$, measured around the $7 \mathrm{MRI}$ devices

Measurements of the magnetic flux density of magnetic fields in the frequency range $8 \div 300 \mathrm{kHz}$ are shown in Figure 4. 


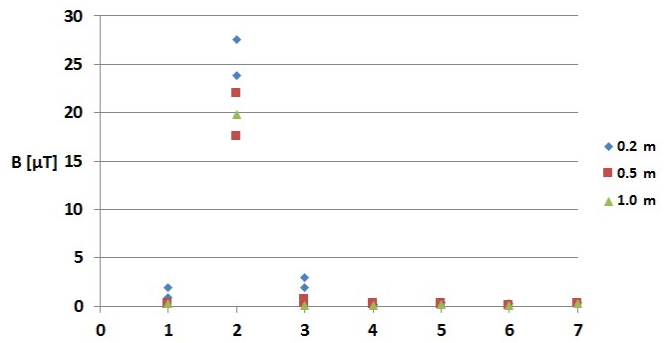

Figure 4. Measured values of the magnetic flux density in the frequency range $8 \div 300 \mathrm{kHz}$ measured around the $7 \mathrm{MRI}$

Measurements of the magnetic field in the two frequency ranges show that, despite the large number of statistically "homogeneous" results, there are also those that deviate greatly. We assume that the reason for them is the type of screening of the specific MRI, design features, as well as the location of the MRI in the procedure shielded room.

As it could be expected the electric field strengths in ELF and LF range were very low, close to the sensitivity of the equipment, so they are not presented here and not commented further.

\subsection{Radiofrequency magnetic field}

Research data from measurements and modelling radio frequency magnetic field inside the bore show [4] that the values are 100 times higher than at the entrance to the bore or at a distance from it. Models for estimating the specific absorption rate (SAR), which should be provided in the MRI documentation, are most often used to estimate the RF magnetic field. The registered values outside the bore gentry depend on the model of the equipment, shielding, the performed procedure - sequences, relaxation time. In this sense, significant values of the radio frequency magnetic field outside the bore are not expected at the places where stay of the personnel is possible. The SAR values that should be included in the equipment documentation may serve as a basis for development of patient and personnel protection approaches.

In Figure 5, data for RF electric fields for the 7 MRI devices are presented.

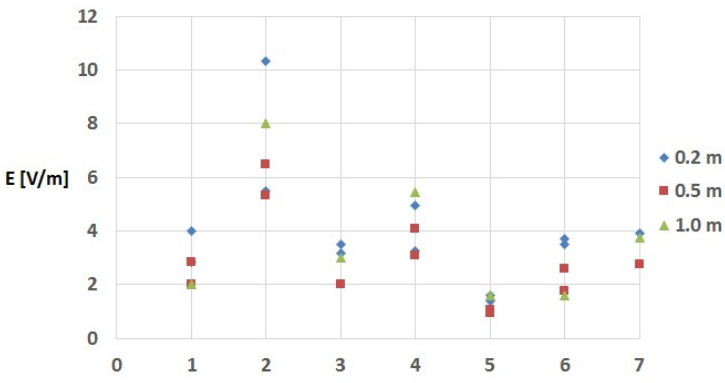

Figure 5. Measured values of the electric field strength of RF field measured around the 7 MRI

\section{Discussion}

There are two aspects to be considered when using magnetic resonance imaging (MRI) equipment for diagnosis: patient and personnel protection.

\subsection{In regard to patient protection}

Prior to the examination, preliminary information for each patient is collected about the health condition, the presence of active and passive medical implants and other medical devices worn on the body, as well as performed interventions of the body that may affect the magnetic resonance imaging examination (tattoos, piercings, etc.). Patients declare presence of such implants or ferromagnetic objects. For women, the fact if they are currently pregnant is important. All these issues are included in a declaration of informed consent, which allows preliminary screening of persons who have contraindications for presence in the room with MRI or conducting the specific study.

The "risk-benefit" principle, which is fundamental to any medical procedure, applies to patients as to the procedures using ionizing radiation. For patients, regardless of the length of the MRI procedure, the risk of exposure to non-ionizing radiation is significantly lower than the benefit of the diagnostic method.

In addition to the approaches described above, to avoid sensory effects in patients undergoing diagnostic procedures with MRI, ICNIRP, HPA, Great Britain [2, $3,4]$ and other organizations recommend slow movement of patients in the gentry. The scientifically proven threshold for vertigo induced by motion in a strong magnetic field is about 1 T. $\mathrm{s}^{-1}$ for more than $1 \mathrm{~s}$. [2]. It is believed that avoiding these sensations will provide protection against other effects of induced electric fields as a result of movement in a static magnetic field.

\subsection{In regard to personnel protection}

Staff protection is a much more complex issue. Often during diagnostic procedures, especially when they are not routine, it is necessary for medical personnel to stay in the shielded room. Exposure to non-ionizing radiation is intermittent, with different durations depending on the type of diagnostic procedure. According to the scientific literature [4], diagnostic procedures can take from about 30 minutes to 90 minutes. It is possible to move several times in the inhomogeneous field during the day for each of the patients.

Different procedures involve different risks of exceeding the exposure limit values. At the same time, the personnel are exposed to noise emitted by switching on/off the coils generating gradient fields. To avoid sensory effects for the staff, it is also recommended to limit the speed of movement in the shielded room.

For both patients and staff, this is a parameter that is difficult to define and control in the shielded room.

The risks for persons who are not directly related to the diagnostic procedures - maintenance of the equipment and hygiene and disinfection of the premises and equipment - should not be neglected. This requires the use of different approaches to personnel exposure and risk assessment.

The limitations that are imposed in relation to patient and personnel safety are related to the projectile effect and its impact on implants on the human body.

The performed measurements of the magnetic flux densities of the static magnetic field show that the 
exposure limit values (ELVs) are not exceeded at the workplaces in the shielded and in the control room, according to the requirements of Directive 2013/35/EU [7].

The action value for magnetic flux density of static magnetic field for interference with active implanted devices, e.g. cardiac pacemakers $-0.5 \mathrm{mT}$ is exceeded at all measuring points in a shielded room, but are not exceeded outside the shielded room, according to the requirements of the Directive 2013/35/EU [7]. The action value for the risk of attraction and projectile risk for static magnetic field - $3 \mathrm{mT}$ is exceeded at all measuring points in the shielded room.

The values of the magnetic flux densities in the ELF and LF range at all points of measurement do not exceed the action values for non-thermal and thermal effects, according to Directive 2013/35/EU which mean that the health effects ELVs and the sensory effects ELVs are kept.

The reference values for static magnetic field according to Recommendation 1999/519/EC [8] are exceeded at all measuring points in the shielded room, which means that there is a risk to the health of workers at specific risk (wearing active and passive implants; wearing medical devices on their bodies (pumps), as well as for pregnant women). In the close vicinity of bore values of ELF and LF magnetic flux density exceed the reference levels according to the Council recommendation as well.

Notwithstanding the demonstrated compliance with the static magnetic field exposure limit values, the risk to medical personnel operating MRI facilities is high due to the need for temporary residence in the shielded room.

On the basis of results of the measurements, as well as the data from the research and the documents of the international organizations, recommendations have been made for the protection of the personnel in the studied MRI departments. These include staff training, restriction of stay and movement in the shielded room, restriction of access for persons at specific risk, health surveillance, etc.

The specifics of the work in MRI shows the impossibility to make an adequate exposure and risk assessment on the basis of only conservative measurement and assessment in accordance with the established exposure limit values and action levels. The introduced limit values do not take into account the duration of exposure, but it should be borne in mind that longer exposure times can lead to health problems, errors and incidents (as a result of fatigue, induced currents in the body).

The risk of inducing electric fields and currents in the body should be considered here. It is not related to the duration of the exposure, but to the spatial distribution of the field in the room.

In fact, there are no standards for assessing the exposure of workplaces with MRI equipment, which is evident from the temporal derogations introduced in Directive 2013/35/EU. The European standard in force [11] shows demonstration of compliance by calculations or design review, but considers MRI mainly as a device. It requires the locations with highest spatial gradient of the static field and controlled access area with values higher than $0.5 \mathrm{mT}$ to be indicated by the manufacturers of the MRI equipment.

However, the derogations introduced in the Directive do not mean that the personnel are protected. The adopted limit values do not reflect the specifics of the personnel exposure in these departments. In this situation, it is not clear which technology is more dangerous for the staff - CT or MRI, since all the necessary protective measures have been taken for the staff servicing the CT, so that there is not even minimal exposure of the workers.

Historically, the development of static magnetic field exposure limits has undergone significant changes over the years. Most of them are caused by the inability to respond to the specifics of different sources of static magnetic field, providing adequate protection. Until 2009, the ACGIH TLV [12], for example, assumed a value for 8-hours exposure with static magnetic field of $60 \mathrm{mT}$ and a ceiling value of $2 \mathrm{~T}$ for whole-body exposure and $8 \mathrm{~T}$ for local exposure. Subsequently, the value for 8 hours disappears, leaving only the ceiling value, compliance with which does not guarantee avoidance of induction of an electric field in the body. This raises the question of whether this change in limit values was not necessary in order to implement the relevant new recommendations and standards from ICNIRP and IEEE, which do not cover the exposure conditions of personnel working with MRI facilities.

\section{CONCLUSION}

The results of measurement of EMFs on workplaces in the vicinity of $1.5 \mathrm{~T}$ MRI equipment show that there are not values above ELVs and Als according to the Directive 2013/35/EU. This is valid for the static magnetic fields, low frequency fields (up to $300 \mathrm{kHz}$ ) and for RF fields. Measured values of static magnetic field are above the Als both for persons in specific risk and for projectile risk.

The MRI technology is safer for the patient than those using ionizing radiation, but this is not the case for the personnel. The directive "does not work" for these sources and those working with them may be not sufficiently protected. There are also other working places that are not sufficiently protected by the Directive - for example, induction sealers and heaters, $5 \mathrm{G}$ technology and others, where exposures to EMF are intermittent; to complex fields; in combination with other physical factors as noise, vibration, and ionizing radiation.

In our opinion, it will be useful for these technologies and workplaces cited above specific requirements based on the Directive to be developed (including MRI).

Regardless of the protection offered / used during diagnostic procedures, attention should also be paid to the acoustic noise generated by the gradient system.

\section{REFERENCES}

1. ICNIRP Guidelines on limits of exposure to static magnetic fields, ICNIRP Publication - 2009, ICNIRP, Oberschleissheim, Germany, 2009. Retrieved from: 
https://www.icnirp.org/cms/upload/publications/ICNI RPstatgdl.pdf

Retrieved on: Mar. 29, 2021

2. ICNIRP Guidelines for limiting exposure to electric fields induced by movement of the human body in a static magnetic field and by time varying magnetic fields below $1 \mathrm{~Hz}$, ICNIRP Publication - 2014, ICNIRP, Oberschleissheim, Germany, 2014.

Retrieved from:

https://www.icnirp.org/cms/upload/publications/ICNI RPmvtgdl 2014.pdf

Retrieved on: Mar. 29, 2021

3. ICNIRP statement on medical magnetic resonance (MR) procedures: protection of patients, ICNIRP Publication - 2004, ICNIRP, Oberschleissheim, Germany, 2004

Retrieved from:

https://www.icnirp.org/cms/upload/publications/ICNI RPMR2004.pdf

Retrieved on: Mar. 29, 2021

4. Protection of Patients and Volunteers Undergoing MRI Procedures: Advice from the Health Protection Agency, Documents of the Health Protection Agency, Radiation, Chemical and Environmental Hazards RCE-7, HPA, London, UK, 2008.

Retrieved from:

https://assets.publishing.service.gov.uk/government/u ploads/system/uploads/attachment data/file/329364/ Protection of patients and volunteers undergoing MRI procedures.pdf

Retrieved on: Feb. 8, 2021

5. H. Kromhout et al., "ICNIRP Statement on diagnostic devices using non-ionizing radiation: Existing regulations and potential health risks," Health Phys., vol. 113, no. 2, pp. 149 - 150, Aug. 2017.

DOI: $10.1097 /$ HP.0000000000000686

PMid: 28658061

6. S. Bongers, P. Slottje, H. Kromhout, "Development of hypertension after long-term exposure to static magnetic fields among workers from a magnetic resonance imaging device manufacturing facility," Environ. Res., vol. 164, pp. 565 - 573, Jul. 2018.

DOI: 10.1016/j.envres.2018.03.008

PMid: 29621724

7. The European Parliament and the Council of the European Union. (Jun. 26, 2013). Directive 2013/35/EC of the European Parliament and of the Council on the minimum health and safety requirements regarding the exposure of workers to the risks arising from physical agents (electromagnetic fields).
Retrieved from:

https://eur-

lex.europa.eu/LexUriServ/LexUriServ.do?uri=OJ:L:20

13:179:0001:0021:EN:PDF

Retrieved on: Dec. 14, 2020

8. The Council of European Union. (Jul. 12, 1999). Council Recommendation 1999/519/EC on the limitation of exposure of the general public to electromagnetic fields (o $\mathrm{Hz}$ to $300 \mathrm{GHz}$ ).

Retrieved from:

https://op.europa.eu/en/publication-detail/Lpublication/9509b04f-1dfo-4221-bfa2c7af77975556/language-en

Retrieved on: Dec. 14, 2020

9. Electromagnetic Fields, vol. 1, Non-binding guide to good practice for implementing Directive 2013/35/EU, European Commission, Brussels, Belgium, 2015.

Retrieved from:

http://bookshop.europa.eu/en/non-binding-guide-togood-practice-for-implementing-directive-2013-35-euelectromagnetic-fields-pbKE0415140/

Retrieved on: Dec. 14, 2020

10. Министерство на труда и социалната политика/Министерство на здравеопазването. (Ное. 26, 2016). Държавен вестник брой 95. Наредба № РД-о7-5 от 15 ноември 2016 г. за минималните изисквания за осигуряване на здравето и безопасността на работещите при рискове, свързани с експозиция на електромагнитни полета.

(Ministry of Labor and Social Policy/Ministry of Health. (Nov. 26, 2016). State gazette No. 95. Ordinance No. RD-O7-5 of 15 November 2016 for the minimal requirements for providing health and safety at work at risks by exposure to electromagnetic fields.) Retrieved from: http://dv.parliament.bg/DVWeb/broeveList.faces\# Retrieved on: Dec. 14, 2020

11. Medical electrical equipment - Part 2-33: Particular requirements for the basic safety and essential performance of magnetic resonance equipment for medical diagnosis, IEC 60601-2-33:2010, Mar. 10, 2010.

Retrieved from:

https://webstore.iec.ch/publication/2647

Retrieved on: Jan. 11, 2021

12. Threshold Limit Values for Chemical Substances and Physical Agents and Biological Exposure Indices, ACGIH, Cincinnati (OH), USA, 2008. 